\title{
Thermal Stability of Globins: Implications of Flexibility and Heme Coordination Studied by Molecular Dynamics Simulations
}

\author{
Laia Julió Plana, ${ }^{\dagger}$ Alejandro D. Nadra, ${ }^{\dagger}$ Dario A. Estrin, ${ }^{\dagger}$ F. Javier Luque, ${ }^{\S} \|_{\odot}$ and Luciana Capece ${ }^{*}{ }^{\dagger} \odot$ \\ ${ }^{\dagger}$ Departamento de Química Inorgánica, Analítica y Química Física, Facultad de Ciencias Exactas y Naturales, Universidad de Buenos \\ Aires/Instituto de Química Física de los Materiales, Medio Ambiente y Energía (INQUIMAE-CONICET), C1428EGA Buenos \\ Aires, Argentina \\ ${ }^{\ddagger}$ Departamento de Fisiología y Biología Molecular y Celular, Facultad de Ciencias Exactas y Naturales, Universidad de Buenos Aires, \\ Departamento de Química Biológica, Facultad de Ciencias Exactas y Naturales, Universidad de Buenos \\ Aires/IQUIBICEN-CONICET, C1428EGA Buenos Aires, Argentina \\ ${ }^{\S}$ Department of Nutrition, Food Sciences and Gastronomy, Faculty of Pharmacy and Food Sciences, University of Barcelona, \\ Campus Torribera, 08921 Santa Coloma de Gramenet, Spain \\ "Institute of Biomedicine (IBUB) and Institute of Theoretical and Computational Chemistry (IQTCUB), University of Barcelona, \\ 08028 Barcelona, Spain
}

Supporting Information

ABSTRACT: Proteins are sensitive to temperature, and abrupt changes in the normal temperature conditions can have a profound impact on both structure and function, leading to protein unfolding. However, the adaptation of certain organisms to extreme conditions raises questions about the structural features that permit the structure and function of proteins to be preserved under these adverse conditions. To gain insight into the molecular basis of protein thermostability in the globin family, we have examined three representative examples: human neuroglobin, horse heart myoglobin, and Drosophila hemoglobin, which differ in their melting temperatures and coordination states of the heme

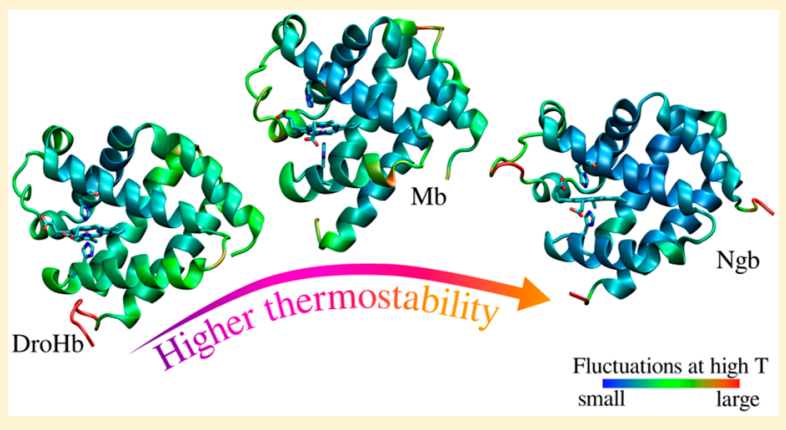
iron in the absence of external ligands. In order to elucidate the possible mechanisms that govern the thermostability of these proteins, microsecond-scale classical molecular dynamics simulations were performed at different temperatures. Structural fluctuations and essential dynamics were analyzed, indicating that the flexibility of the CD region, which includes the two short $\mathrm{C}$ and $\mathrm{D}$ helixes and the connecting CD loop, is directly related to the thermostability. We observed that a larger inherent flexibility of the protein produces higher thermostability, probably concentrating the thermal fluctuations observed at high temperature in flexible regions, preventing unfolding. Globally, the results of this work improve our understanding of thermostability in the globin family.

\section{INTRODUCTION}

Globins constitute a widely distributed family of proteins that are responsible for a great variety of biological functions in all kingdoms of life. ${ }^{1-3}$ They contain heme as a prosthetic group, which is found inserted into the core of the protein, and generally fold in a globular 3-over-3 sandwich motif formed by helices labeled A, B, E, F, G, and H (Figure 1A). The remaining short helices, $\mathrm{C}$ and $\mathrm{D}$, together with the $\mathrm{CD}$ loop, form the $\mathrm{CD}$ region.

The two regions that are delimited by the plane of the porphyrin ring are commonly known as the proximal and distal sites. The iron in the heme group is bonded by four nitrogen atoms of the porphyrin ring in its equatorial coordination sites and a conserved histidine residue in the proximal site (named HisF8 for its position in the F helix), forming the characteristic pentacoordinated state $(5 \mathrm{c})$. On the other hand, the distal site usually binds a small external ligand, such as $\mathrm{O}_{2}, \mathrm{CO}, \mathrm{NO}$, or
$\mathrm{H}_{2} \mathrm{~S}$, which is closely related to protein function. ${ }^{4}$ In some cases, a protein residue coordinates the iron reversibly (especially in the ferrous state) in the distal site, forming a hexacoordinated (6c) protein (Figure 1B). Frequently, the residue that occupies the distal site in $6 c$ globins is a histidine residue in the seventh position of the E helix, HisE7, resulting in a bishistidyl hexacoordinated species. However, residues located in other positions, such as E11, might also coordinate to the iron as observed in other globins (especially truncated hemoglobins ${ }^{5}$ ). This process introduces an additional mechanism to control the function of globins, since binding of exogenous ligands requires cleavage of the $\mathrm{Fe}$-distal residue bond. This finely tuned equilibrium between the $6 \mathrm{c}$ and $5 \mathrm{c}$ states can be experimentally characterized by the equilibrium

Received: November 22, 2018

Published: December 5, 2018 


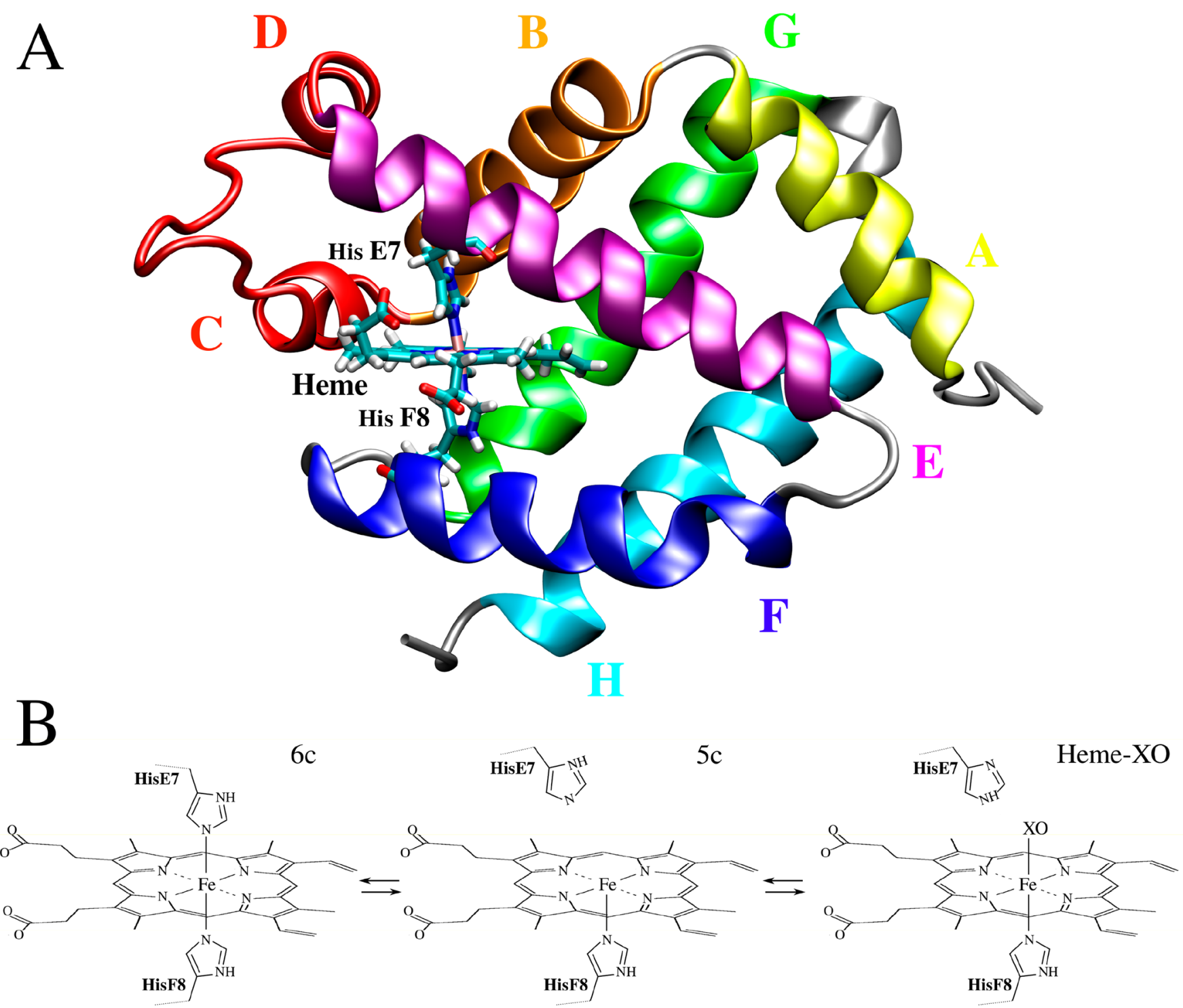

Figure 1. (A) Cartoon representation of the crystal structure of neuroglobin (PDB code 1OJ6) as an example of the conserved 3-over-3 globin fold. Each helix is colored differently and depicted in the figure. (B) $6 \mathrm{c} \rightleftarrows 5 \mathrm{c}$ equilibrium displayed by the heme group in hexacoordinated globins. XO represents a generic small ligand such as $\mathrm{O}_{2}, \mathrm{CO}$, or $\mathrm{NO}$.

constant $K_{\mathrm{His}}=k_{\mathrm{on}, \mathrm{His}} / k_{\mathrm{off,His}}$, where $k_{\mathrm{on}, \mathrm{His}}$ and $k_{\mathrm{off}, \mathrm{His}}$ denote the kinetic constants for His binding and unbinding, respectively. In previous works, ${ }^{6-8}$ we have shown that protein flexibility, especially in the $\mathrm{CD}$ region (Figure $1 \mathrm{~A}$, region in red), is a key factor in the regulation of the $6 \mathrm{c} \rightleftarrows 5 \mathrm{c}$ equilibrium.

It is well-known that proteins are sensitive to environmental conditions, including changes in temperature and pressure, among others. In particular, a rise in temperature can lead to alterations in protein stability and flexibility and even to protein unfolding. However, differential behavior toward temperature is observed even in proteins that display high sequence identity and exhibit very similar three-dimensional structures. This is the case, for instance, with homologous enzymes of thermally adapted and nonadapted organisms. ${ }^{9-11}$ Interestingly, proteins that belong to thermophilic microorganisms are able to retain their structure and function even under high-temperature conditions. ${ }^{12,13}$ Thus, adaptation to extreme temperature is not easy to explain.
From the folding landscape theory, the stability of the native state is governed by the free energy gap between the ensembles of microconfigurations corresponding to the folded and unfolded states. Experimentally, this is commonly related to the melting temperature of the protein. ${ }^{14}$ In this context, denaturation involves a large loss of enthalpic stabilization but, on the other hand, a large gain in entropic stabilization. Thus, a larger number of contacts in the native state, which is typically associated with a greater rigidity of the protein structure, will favor the enthalpic contribution and benefit high thermal stability. ${ }^{15-22}$ On the other hand, in regard to the entropic contribution to the thermal stability, a larger conformational entropy in the folded state, which is typically associated with higher flexibility of the protein structure, will also benefit thermal stability. ${ }^{23}$ Indeed, a more recent work that compares the thermal stabilities of truncated hemoglobins from mesophilic and thermophilic organisms indicates that proteins with higher flexibility display higher thermal stability. ${ }^{24}$ Thus, it 
is not straightforward to predict a priori the effect of protein flexibility on thermal stability.

In this work, we sought to gain insight into the molecular basis of protein thermostability in globins. When focusing on the globin family, the picture is even more complicated because of the presence of the heme group and the different coordination states that the iron in the heme group can adopt. In order to perform this study, we selected three representative examples: human brain neuroglobin $(\mathrm{Ngb})$, horse heart myoglobin ( $\mathrm{Mb})$, and Drosophila hemoglobin (DroHb). These examples differ in their melting temperatures $\left(T_{\mathrm{m}}\right)$ and in their coordination states in absence of external ligands. $\mathrm{Ngb}$ has been reported to have $T_{\mathrm{m}}=373 \mathrm{~K}$ (or $363 \mathrm{~K}$ in the presence of a disulfide bridge in the $\mathrm{CD}$ region) and is the most thermostable of the three proteins. The second example is $\mathrm{Mb}$, with $T_{\mathrm{m}}=354 \mathrm{~K}$, and finally, DroHb has been reported to have $T_{\mathrm{m}}=349 \mathrm{~K}^{25}$ Regarding the $6 \mathrm{c} \rightleftarrows 5 \mathrm{c}$ equilibrium, DroHb populates both coordination states, with $K_{\mathrm{His}}=18 ; \mathrm{Mb}$ displays only the $5 \mathrm{c}$ state, with $K_{\mathrm{His}} \ll 1$; and $\mathrm{Ngb}$ predominantly populates the $6 \mathrm{c}$ state, with $K_{\mathrm{His}}=280 .^{25}$ Originally, the higher thermostability of $\mathrm{Ngb}$ in comparison with $\mathrm{Mb}$ had been assigned to the different coordination state of the heme group, which provides an additional bond between the $\mathrm{Fe}$ in the heme group and a protein residue (HisE7). However, the $T_{\mathrm{m}}$ of $\mathrm{Ngb}$ bound to $\mathrm{CO}$ (where the $\mathrm{Fe}-\mathrm{HisE} 7$ bond is not present) is $368 \mathrm{~K},{ }^{25}$ which, although lower than that of $\mathrm{Ngb} 6 \mathrm{c}$, is still significantly higher than the $T_{\mathrm{m}}$ of $\mathrm{Mb}$. Thus, a more complex mechanism that involves not only the heme and its environment but also the protein matrix is expected to be involved in the thermal stability of globins. The results from this work indicate that in globins, protein flexibility, in particular flexibility of the $C D$ region, is a key factor governing the thermal stability, improving our understanding of thermostability in the globin family.

\section{COMPUTATIONAL METHODS}

Initial Structures. The initial structures of $\mathrm{DroHb}$, horse heart $\mathrm{Mb}$, and human brain $\mathrm{Ngb}$ were obtained from X-ray

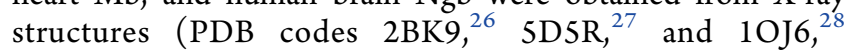
respectively). A superposition of the three structures can be seen in Figure S1. For Ngb and DroHb, the pentacoordinated (5c) structures were obtained from the hexacoordinated (6c) one by release of the $\mathrm{Fe}-\mathrm{HisE7}$ bond constraint followed by a minimization and slow equilibration (as described below). Protonation states of amino acid residues were set to correspond to those at neutral $\mathrm{pH}$. As in previous works, ${ }^{6,8,29,30}$ in the case of histidine amino acids, protonation was established in order to favor $\mathrm{H}$-bond formation, with the exception of HisE7 and HisF8 (i.e., the two histidine residues that coordinate the iron in the heme group), which were protonated at $\mathrm{N}-\delta$.

Molecular Dynamics Simulations. Classical molecular dynamics (MD) simulations were performed by using the PMEMD module of the Amber14 package ${ }^{31}$ in its CUDA version for GPU computing. All of the simulations were run for the ferrous state of the heme iron. The starting structures were immersed in an octahedral box of TIP3P water molecules. ${ }^{32} \mathrm{~A}$ minimum distance of $10 \AA$ from the protein surface to the end of the box was used. Residue parameters correspond to the AMBER ff14SB force field ${ }^{31}$ except for the heme, for which the parameters correspond to those developed ${ }^{33}$ and used in several heme-protein studies. ${ }^{29,34-39}$
All of the simulations were performed using periodic boundary conditions with a $10 \AA$ cutoff for nonbonded interactions in conjunction with the Particle Mesh Ewald (PME) summation method for treating long-range electrostatic interactions. The SHAKE algorithm was employed to keep bonds involving hydrogen atoms at their equilibrium lengths, ${ }^{40}$ which allowed a 2 fs time step for the integration of Newton's equations. All of the simulations were performed in the NPT ensemble using the Berendsen barostat ${ }^{41}$ (in all cases at $1 \mathrm{~atm}$ ) and the Langevin thermostat. ${ }^{42}$ Each hemoglobin was run using three different temperatures for each coordination state of heme ( $5 \mathrm{c}$ and $6 \mathrm{c}$ ): $300 \mathrm{~K}$ (room temperature), $T_{\mathrm{m}}$ for each $\operatorname{protein}^{25}(349,354$, and $373 \mathrm{~K}$ for $\mathrm{DroHb}, \mathrm{Mb}$, and $\mathrm{Ngb}$, respectively), and a common high temperature of $363 \mathrm{~K}$.

Equilibration consisted of an energy minimization of the initial structure followed by a slow heating to the chosen temperature. In order to reach the desired temperature, a temperature ramp of $0.5 \mathrm{~K} / \mathrm{ps}$ was made. All of the production MD simulations were run for $1 \mu \mathrm{s}$. The validity of the TIP3P water model at different temperatures was analyzed by calculating the radial distribution function and the water density at the different simulation temperatures (Table S1, Figure S2, and Table S2). The results indicate that the change in density is consistent with the experimental observations and that globally the solvent structure is conserved at the different temperatures.

Analysis of the MD simulations was done using Visual Molecular Dynamics (VMD) ${ }^{43}$ and the Cpptraj tool from the Amber14 package. ${ }^{31}$ For the MD analysis, the first 100 ns was discarded in order to exclude the trajectory corresponding to the initial temperature equilibration (see Figure S3).

Finally, replicas of selected MD simulations were considered to further check the results. These replicas $(0.5 \mu \mathrm{s})$ were performed for DroHb $6 \mathrm{c}$ at 300 and $363 \mathrm{~K}$ and for Ngb $6 \mathrm{c}$ at 300 and $363 \mathrm{~K}$. The analysis of these MD simulations can be found in Figures S4 and S5 and Table S3. The results obtained are in agreement with the data derived from the $1 \mu \mathrm{s} \mathrm{MD}$ simulations presented here.

Essential Dynamics. To examine the influence of temperature on the dynamical properties of proteins and to capture the large-scale motions sampled along the MD simulations, essential dynamics (ED) analysis was used. ${ }^{44} \mathrm{ED}$ involves the diagonalization of the covariance matrices of atomic positions along the trajectory, yielding the eigenvectors that define the essential motions of the protein. The ED analysis was performed for the backbone atoms in order to isolate the motions associated with large conformational movements of the protein backbone. Additional calculations were performed including all the amino acid side chains, which are shown in Figure S7. The alignment was done considering only the cores of the helices (without the residues in the loops and the three first and three last residues of each helix). However, including terminal residues allows the observation of the onset of denaturation with increasing temperature, so it was done in all cases. In order to analyze the configurational space explored along the MD run, the compositions of selected essential modes were determined by averaging the absolute values of the corresponding eigenvectors for each protein residue. This type of analysis is valuable to explore the structural and dynamical relationships in proteins. ${ }^{6,45,46}$ 


\section{RESULTS}

In order to understand the structural and dynamical features that determine the differential thermostability in globins, we selected three members of the family that display diverse behavior when exposed to high-temperature conditions: $\mathrm{DroHb}, \mathrm{Mb}$, and $\mathrm{Ngb}$ (see above). As noted above, Ngb displays higher thermostability, which was partially assigned to the formation of the $6 \mathrm{c}$ bishistidyl coordination. ${ }^{47}$ However, although DroHb also populates the $6 \mathrm{c}$ state, ${ }^{26}$ its thermostability is even lower than that of $\mathrm{Mb}$, which is present only in the $5 c$ state (in the absence of external ligands ${ }^{25}$ ), indicating that there is no direct connection between thermostability and hexacoordination.

To shed light on the possible factors that govern thermostability in globins, classical MD simulations were run at different temperatures for both the $5 \mathrm{c}$ and $6 \mathrm{c}$ states. A summary of the systems and calculations is provided in Table 1.

Table 1. Summary of the MD Simulations Performed in This Work

\begin{tabular}{|c|c|c|c|c|c|}
\hline & PDB code & $T m(\mathrm{~K})$ & Simulati & & Time \\
\hline \multirow{3}{*}{ DroHb } & \multirow{3}{*}{ 2BK9 } & \multirow{3}{*}{349} & \multirow{3}{*}{$5 \mathrm{c}$ and $6 \mathrm{c}$ at } & \multirow{2}{*}{$\begin{array}{l}300 \mathrm{~K} \\
349 \mathrm{~K}\end{array}$} & $1 \mu \mathrm{s}^{\mathrm{a}}$ \\
\hline & & & & & $1 \mu \mathrm{s}$ \\
\hline & & & & $363 \mathrm{~K}$ & $1 \mu \mathrm{s}^{\mathrm{a}}$ \\
\hline \multirow{3}{*}{$\mathrm{Mb}$} & \multirow{3}{*}{$5 \mathrm{D} 5 \mathrm{R}$} & \multirow{3}{*}{354} & \multirow{3}{*}{$5 c$ and $6 c$ at } & & $1 \mu \mathrm{s}$ \\
\hline & & & & $354 \mathrm{~K}$ & $1 \mu \mathrm{s}$ \\
\hline & & & & $363 \mathrm{~K}$ & $1 \mu \mathrm{s}$ \\
\hline \multirow{3}{*}{ Ngb } & \multirow{3}{*}{$10 J 6$} & \multirow{3}{*}{373} & \multirow{3}{*}{$5 \mathrm{c}$ and $6 \mathrm{c}$ at } & \multirow{3}{*}{$\begin{array}{l}363 \mathrm{~K} \\
373 \mathrm{~K}\end{array}$} & $1 \mu \mathrm{s}^{\mathrm{a}}$ \\
\hline & & & & & $1 \mu \mathrm{s}^{\mathrm{a}}$ \\
\hline & & & & & $1 \mu \mathrm{s}$ \\
\hline
\end{tabular}

${ }^{a}$ An additional replica $(0.5 \mu \mathrm{s})$ was run as an additional test.
At room temperature, all of the structures remained stable along the MD simulations, as observed in the results of the root-mean-square deviation (RMSD) calculations using the original X-ray structure as the reference (Figure S3). However, with increasing temperature, important differences were observed among the different proteins. DroHb (5c and $6 c$ ) at room temperature displays an RMSD of $\sim 1 \AA$, a regular value for the RMSD of a stable protein. With the increase in temperature this value is maintained for $\sim 400 \mathrm{~ns}$ for the $5 \mathrm{c}$ coordination state and $200 \mathrm{~ns}$ for the $6 \mathrm{c}$ one. At these points, a sudden change is observed, which suggests that $\mathrm{DroHb}$ starts to lose its tertiary structure. $\mathrm{Mb} 5 \mathrm{c}$ also displays an RMSD of $\sim 1 \AA$ at room temperature. With the increase in temperature, the average RMSD value increases to $\sim 2 \AA$ and is maintained along the rest of the simulation. This is probably an indication of a more stable state in $\mathrm{Mb}$ compared with $\mathrm{DroHb}$. $\mathrm{Ngb}$, on the other hand, displays an RMSD value of $\sim 2 \AA$ at room temperature. With the increase in temperature this value is maintained, consistent with the high thermostability of $\mathrm{Ngb}$. The RMSD results are very similar for both coordination states. Taken together, these results indicate that $\mathrm{DroHb}$ is the globin protein that displays the largest deviation from the native structure under high-temperature conditions, followed by $\mathrm{Mb}$. On the other hand, Ngb does not show significant structural alterations from the initial structure at the different temperatures.

The flexibility of the different proteins was initially evaluated by calculating the RMSD values along the MD trajectory using the time-averaged structure as the reference. This approach has been used in previous works as a comparative measure of protein flexibility. ${ }^{6,48,49}$ The average RMSD values were obtained for the whole protein and for selected regions in the $5 c$ and $6 c$ coordination states (Table 2). The lowest global RMSD value at room temperature is observed for $\mathrm{DroHb}$ in both the $5 c$ and $6 c$ states. Interestingly, for $\mathrm{Ngb} 6 \mathrm{c}$ this value remains similar at high temperature, as the difference between the RMSD values at 363 and $300 \mathrm{~K}\left(\Delta \mathrm{RMSD}_{363-300 \mathrm{~K}}\right)$ amounts to $0.2 \AA$, demonstrating that increasing the temperature does not significantly alter the average mobility. The increase in temperature causes a large increase in the RMSD value of DroHb $5 \mathrm{c}\left(\Delta \mathrm{RMSD}_{363-300 \mathrm{~K}}=1.8 \AA\right)$ and moderate increases for $\mathrm{DroHb} 6 \mathrm{c}\left(\Delta \mathrm{RMSD}_{363-300 \mathrm{~K}}=1.0\right)$ and $\mathrm{Mb} 5 \mathrm{c}$ $\left(\Delta \mathrm{RMSD}_{363-300 \mathrm{~K}}=0.8 \AA\right)$.

Table 2. Average RMSDs and Standard Deviations (in $\AA$ ) for DroHb, Mb, and Ngb in Both Coordination States at Different Temperatures along the MD Simulations ${ }^{a}$

\begin{tabular}{|c|c|c|c|c|c|c|c|c|c|c|}
\hline & & \multicolumn{3}{|c|}{ DroHb } & \multicolumn{3}{|c|}{$\mathrm{Mb}$} & \multicolumn{3}{|c|}{$\mathrm{Ngb}$} \\
\hline & & $300 \mathrm{~K}$ & $349 \mathrm{~K}$ & $363 \mathrm{~K}$ & $300 \mathrm{~K}$ & $354 \mathrm{~K}$ & $363 \mathrm{~K}$ & $300 \mathrm{~K}$ & $363 \mathrm{~K}$ & $373 \mathrm{~K}$ \\
\hline \multirow{4}{*}{$5 c$} & whole protein & $0.8 \pm 0.1$ & $1.6 \pm 0.3$ & $2.6 \pm 0.5$ & $1.2 \pm 0.2$ & $2.1 \pm 0.2$ & $2.0 \pm 0.3$ & $1.4 \pm 0.2$ & $2.1 \pm 0.4$ & $2.4 \pm 0.4$ \\
\hline & $\mathrm{CD}$ region & $1.0 \pm 0.3$ & $1.2 \pm 0.3$ & $1.7 \pm 0.4$ & $1.3 \pm 0.3$ & $1.9 \pm 0.6$ & $2.9 \pm 0.6$ & $1.5 \pm 0.2$ & $3.0 \pm 1.2$ & $3.1 \pm 1.1$ \\
\hline & terminal residues & $0.9 \pm 0.2$ & $3.4 \pm 0.9$ & $5.5 \pm 1.6$ & $1.6 \pm 0.3$ & $2.1 \pm 0.6$ & $2.3 \pm 0.4$ & $2.9 \pm 0.6$ & $3.2 \pm 0.8$ & $4.5 \pm 0.8$ \\
\hline & EF loop & $1.2 \pm 0.8$ & $1.6 \pm 0.7$ & $3.8 \pm 0.9$ & $1.2 \pm 0.3$ & $2.2 \pm 0.5$ & $2.2 \pm 0.5$ & $1.0 \pm 0.3$ & $1.3 \pm 0.3$ & $1.4 \pm 0.4$ \\
\hline \multirow{4}{*}{$6 c$} & whole protein & $1.3 \pm 0.1$ & $2.1 \pm 0.5$ & $2.3 \pm 0.5$ & $1.8 \pm 0.2$ & $3.2 \pm 0.4$ & $2.9 \pm 0.5$ & $1.6 \pm 0.3$ & $1.8 \pm 0.3$ & $1.9 \pm 0.3$ \\
\hline & $\mathrm{CD}$ region & $1.1 \pm 0.3$ & $1.5 \pm 0.6$ & $1.5 \pm 0.4$ & $1.8 \pm 0.5$ & $2.4 \pm 0.6$ & $2.0 \pm 0.4$ & $2.4 \pm 0.8$ & $2.9 \pm 0.6$ & $3.0 \pm 0.8$ \\
\hline & terminal residues & $1.2 \pm 0.3$ & $4.2 \pm 1.3$ & $4.8 \pm 1.5$ & $2.3 \pm 0.5$ & $6.7 \pm 1.3$ & $5.6 \pm 1.1$ & $2.9 \pm 0.6$ & $2.8 \pm 0.9$ & $3.2 \pm 0.9$ \\
\hline & EF loop & $3.2 \pm 0.8$ & $3.6 \pm 1.4$ & $2.4 \pm 0.8$ & $2.8 \pm 0.6$ & $4.4 \pm 1.1$ & $5.4 \pm 1.3$ & $1.4 \pm 0.4$ & $1.3 \pm 0.4$ & $1.2 \pm 0.5$ \\
\hline
\end{tabular}

${ }^{a}$ RMSD values were computed using the whole protein excluding the terminal residues (the first and last 10 residues), only the CD region (residues 37 to 54 for $\mathrm{DroHb}, 37$ to 57 for $\mathrm{Mb}$, and 36 to 55 for $\mathrm{Ngb}$ ), only the terminal residues (the first and last 10 residues), and only the EF loop (residues 76 to 80 for DroHb, 77 to 82 for $\mathrm{Mb}$, and 78 to 81 for $\mathrm{Ngb}$ ). RMSDs were calculated only for the backbone heavy atoms (C, C $\alpha, \mathrm{N}$, O) using the time-averaged structure as the reference. To facilitate the comparison, RMSD values between 0 and $2 \AA$ are shown in plain text, those between 2 and $3 \AA$ in italics, and those higher than $3 \AA$ in bold. 
I)

I)
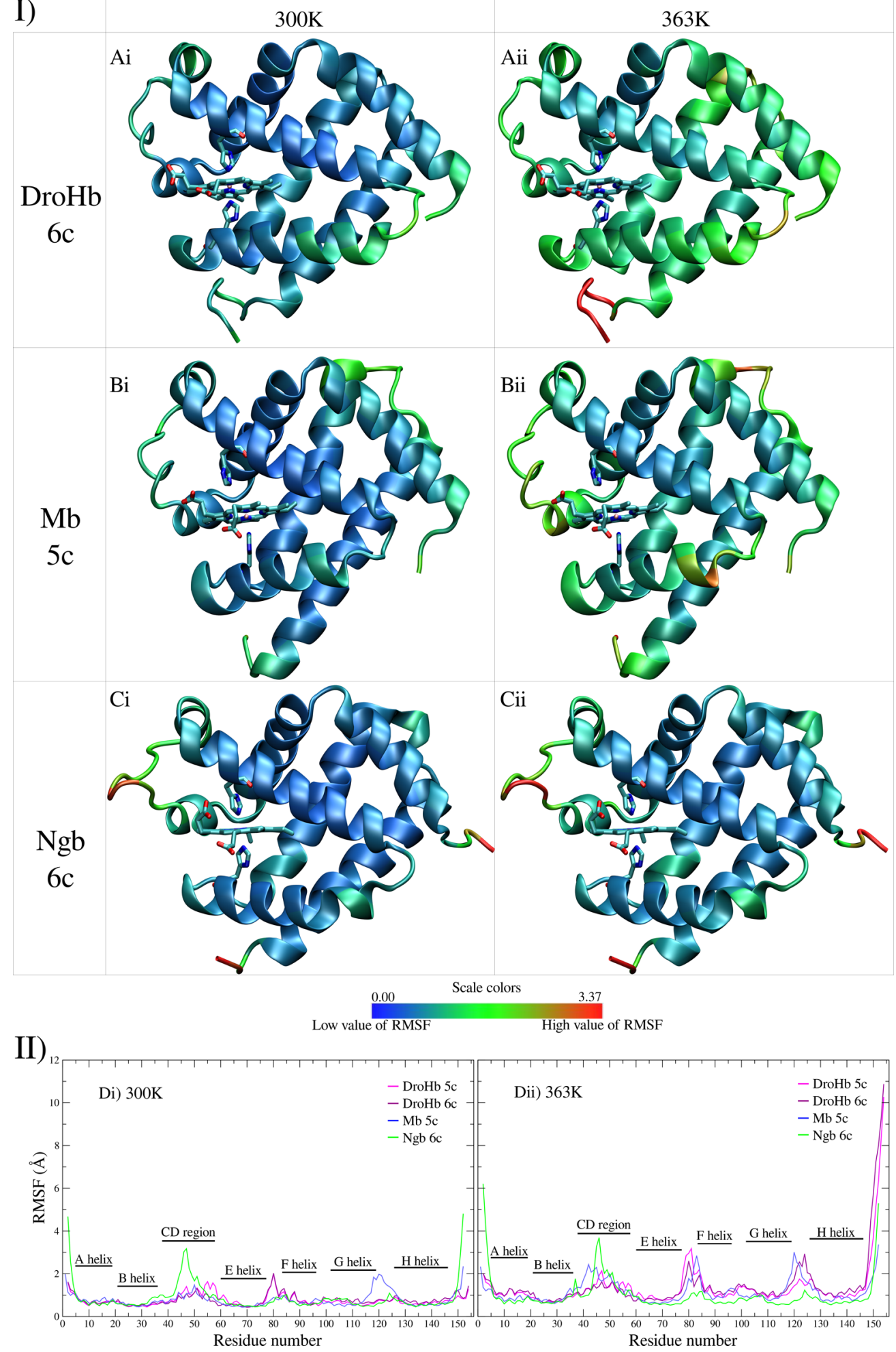

Figure 2. (I) Time-averaged RMSFs (in $\AA$ ) of the backbone heavy atoms along the protein structure observed in MD trajectories (RMSF results per residue are given in Figure S6). For each protein, the most relevant coordination state is shown, namely, $5 \mathrm{c}$ for $\mathrm{Mb}, 6 \mathrm{c}$ for $\mathrm{Ngb}$, and $6 \mathrm{c} \mathrm{for}$ $\mathrm{DroHb}$ (similar results were found for the DroHb 5c species). Results are shown on a BGR color scale: blue, green, and red stand for regions with low, intermediate, and high mobility. (Ai) DroHb 6c at $300 \mathrm{~K}$. (Aii) DroHb 6c at $363 \mathrm{~K}$. (Bi) Mb 5c at $300 \mathrm{~K}$. (Bii) Mb 5c at $363 \mathrm{~K}$. (Ci) Ngb 6c at $300 \mathrm{~K}$. (Cii) Ngb 6c at $363 \mathrm{~K}$. (II) Comparison of the RMSF results at (Di) $300 \mathrm{~K}$ and (Dii) $363 \mathrm{~K}$ for DroHb 5c (magenta), DroHb 6c (purple), Mb 5c (blue), and Ngb 6c (green).

Additionally, Ngb shows high RMSD values for the terminal residues at room temperature, but these values do not increase when the temperature is raised $\left(\Delta \mathrm{RMSD}_{363-300 \mathrm{~K}}=-0.1 \AA\right.$ for 6c and $0.3 \AA$ for 5c). On the other hand, DroHb shows large increases in the RMSDs of the terminal residues in both coordination states, which is probably an indication of the 


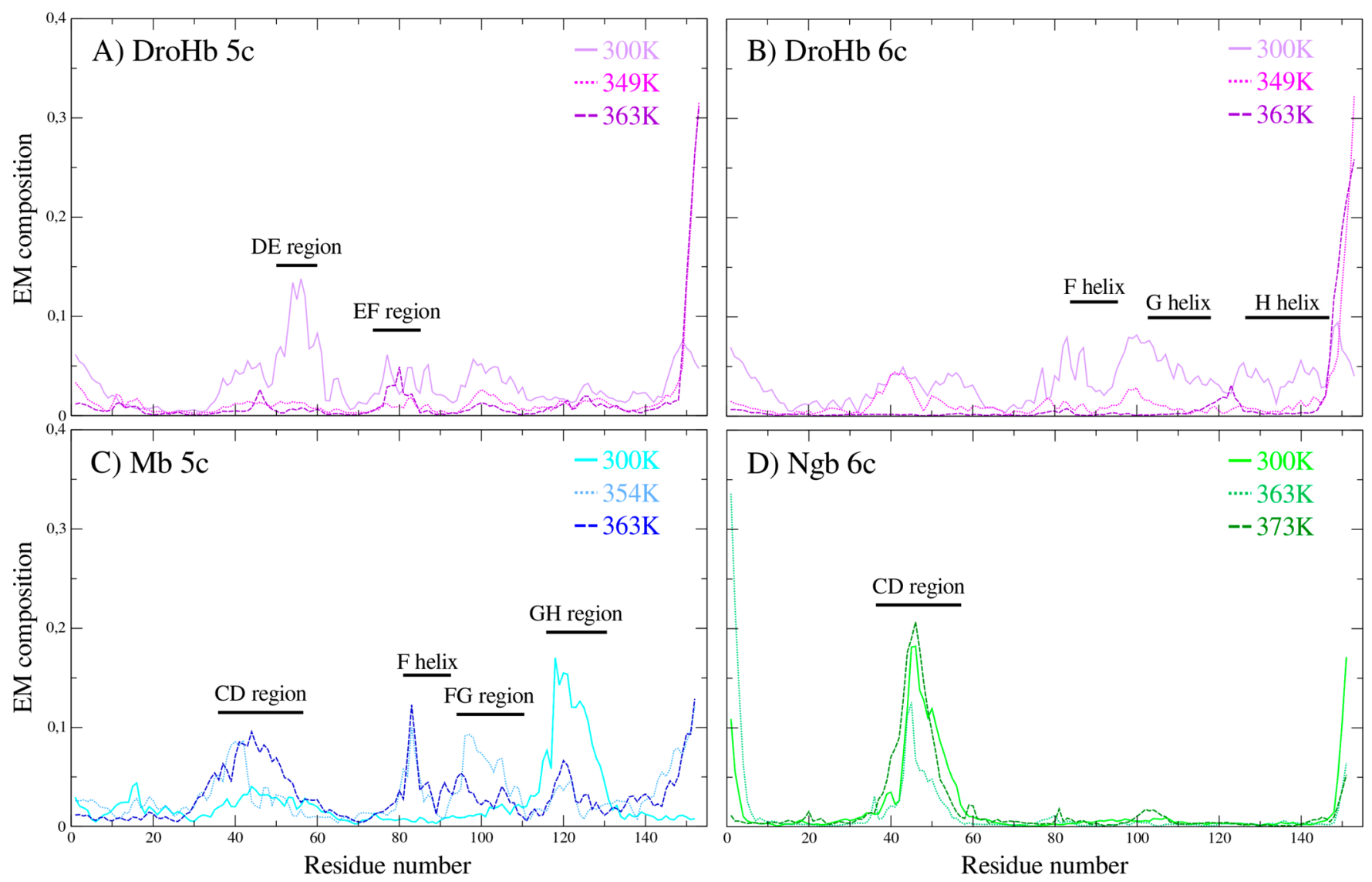

Figure 3. Compositions of the principal essential modes as functions of the residue number. Results at room temperature, at each protein's $T_{\mathrm{m}}$, and at $363 \mathrm{~K}$ are displayed with solid, dashed, and dotted lines, respectively. (A) DroHb 5c at 300, 349, and $363 \mathrm{~K}$. (B) DroHb 6c at 300, 349 , and 363 K. (C) Mb 5c at 300, 354, and $363 \mathrm{~K}$. (D) Ngb 6c at 300, 363, and $373 \mathrm{~K}$. Additionally, Figure S7 also shows comparisons of EM compositions of the weighted averages of the first five EMs and of the EMs calculated for all atoms including the side chains. In both cases, no significant differences were observed with the data presented in this figure.

onset of the unfolding process $\left(\Delta \mathrm{RMSD}_{363-300 \mathrm{~K}}=3.6 \AA\right.$ for DroHb $6 \mathrm{c}$ and $4.6 \AA$ for $5 \mathrm{c}$ ).

When comparing the movement of the $\mathrm{CD}$ region, $\mathrm{Ngb}$ shows an increase in the RMSD value when the temperature is increased $\left(\Delta \mathrm{RMSD}_{363-300 \mathrm{~K}}=0.5 \AA\right.$ for $6 \mathrm{c}$ and $1.5 \AA$ for $\left.5 \mathrm{c}\right)$, which is not observed in DroHb. In $\mathrm{Mb}$, an increase in the mobility of the CD region is also observed. Finally, the EF loop shows low mobility in $\mathrm{Ngb}$, a trait maintained at high temperature, while in $\mathrm{Mb} 5 \mathrm{c}$ there is a large increase of the mobility of this region

When the $5 c$ and $6 c$ states are compared in each case, no significant differences are observed with respect to their behavior toward temperature variations. In $\mathrm{DroHb}$, both coordination states show an increase of $\sim 4 \AA$ in the RMSD value for the terminal residues, which is the most important variation for this protein. Although the $5 \mathrm{c}$ and $6 \mathrm{c}$ forms of DroHb do not show the same absolute RMSD values, they exhibit similar tendencies with increasing temperature. In the $\mathrm{Ngb}$ case, the temperature trend is also conserved between the $5 c$ and $6 c$ states, and the RMSD results for the different protein regions are very similar. In regard to $\mathrm{Mb}$, the behavior of the $6 c$ and $5 c$ states is similar, although the absolute values in $6 \mathrm{c} \mathrm{Mb}$ are larger, consistent with the fact that the $6 \mathrm{c}$ state is less stable.

Apart from RMSD calculations, the snapshots sampled in MD simulations were analyzed in detail using root-meansquare fluctuation (RMSF) calculations, ED analysis, and conformational entropy calculations. Finally, the evolution of the native contacts along the trajectories was also examined to gain information on the differential thermostability of each of the studied systems.

Figures 2 and S6 show the RMSFs obtained for each protein at room and high temperature. As can be observed, the mobility of the protein is increased with the rise in temperature in all cases. In the $\mathrm{DroHb}$ case, at room temperature (Figure 2Ai) the protein shows low mobility, except for the C-terminal residues (147 to 153 ) and the $\mathrm{N}$-terminus of the $\mathrm{F}$ helix. Increasing the temperature causes an increase in mobility all over the protein (Figure 2Aii). The C-terminus (specifically residues 147 to 153 ) is significantly altered, as indicated by its RMSF value, which goes from about 1 to $10 \AA$, an increase that shows the onset of the denaturation process. The $\mathrm{B}$ and $\mathrm{E}$ helices are the regions with the lowest variations. Interestingly, the $\mathrm{CD}$ region barely increases its mobility at high temperature.

On the other hand, $\mathrm{Mb}$, unlike $\mathrm{DroHb}$, does not show a significant increase in the mobility of the $\mathrm{N}$ - and C-terminal residues at high temperature. However, regions that are rigid at room temperature increase their fluctuations at high temperature. At room temperature (Figure $2 \mathrm{Bi}$ ), the $\mathrm{C}$-terminal side of the $\mathrm{G}$ helix is the region with the highest mobility, followed by the $\mathrm{CD}$ region and the $\mathrm{GH}$ loop. When the temperature is increased (Figure 2Bii), regions that show rigidity at room temperature, such as the $\mathrm{N}$-terminal zone of the $\mathrm{F}$ helix and the 

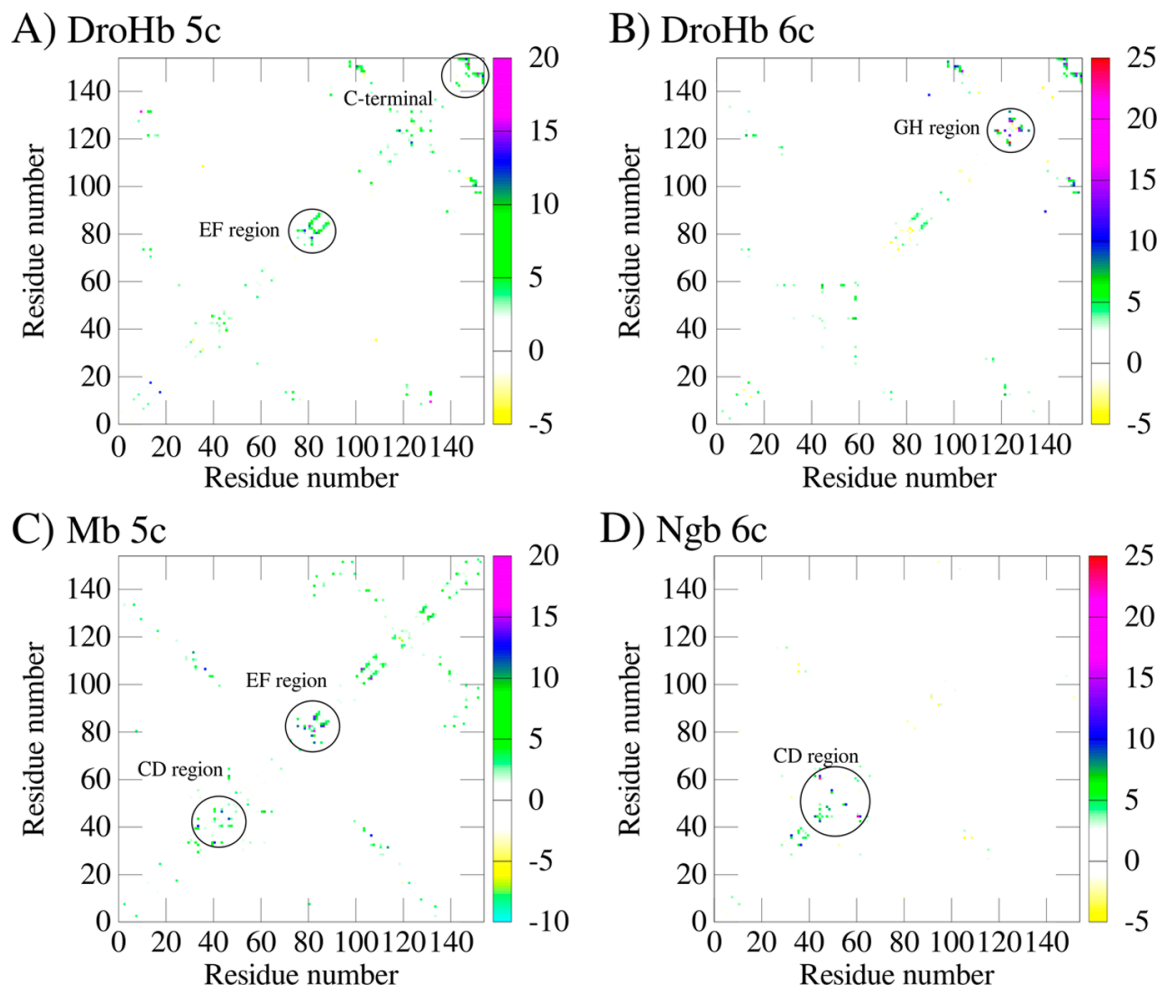

Figure 4. Differential contact maps from the MD simulations at 300 and $363 \mathrm{~K}$ for (A) DroHb 5c, (B) DroHb 6c, (C) Mb 5c, and (D) Ngb 6c. Values were obtained by calculating the number of contacts at $300 \mathrm{~K}$ minus the number of contacts at $363 \mathrm{~K}$ for each residue. Contacts were calculated using Cpptraj ${ }^{31}$ using a cutoff of $5.0 \AA$ A.

C-terminal region of the $\mathrm{G}$ helix, display high fluctuations. Additionally, the mobility of the $\mathrm{CD}$ region increases slightly, but to a lesser extent compared with the regions mentioned above.

$\mathrm{Ngb}$ (Figure 2Ci,Cii) shows practically no variations in the structural fluctuations when the temperature is increased. The same regions with reduced mobility at room temperature remain almost unaltered at high temperature. The regions that show slight increases in mobility are the $\mathrm{N}$ - and C-terminal regions, the $\mathrm{C}$-terminus of the $\mathrm{A}$ helix, the $\mathrm{CD}$ region, and the N-termini of the F, G, and E helices. Finally, Figure 2Di,Dii compares the time-averaged RMSFs for each protein residue at 300 and $363 \mathrm{~K}$. We observe that the structured regions display low mobility at room temperature in the three cases. Increasing the temperature has a minor effect on the RMSF profile of $\mathrm{Ngb}$, with the largest fluctuations being limited to the $\mathrm{CD}$ region together with the $\mathrm{N}$ - and C-terminus. On the other hand, in $\mathrm{DroHb}$ and $\mathrm{Mb}$ we not only observe an increase in the mobility of the $\mathrm{GH}$ and $\mathrm{EF}$ loops, respectively, but also increased fluctuations of the structured regions (especially helices A, F, and G in DroHb and F and G in Mb).

ED calculations permit identification of the large-amplitude motions explored along the MD simulations. The first essential mode (EM) explains $17-27 \%$ of the structural variance at 300 $\mathrm{K}$ for the different states of the three globins, whereas its contribution increases to $19-52 \%$ at the highest temperature (Table S4). In contrast, the second EM varies between 9 and $18 \%$ over the whole range of temperature for all of the systems. It is also worth noting that the contribution of the two first eigenvectors is little affected by temperature in $\mathrm{Ngb}$, whereas their contributions to the structural variance are more influenced by temperature in $\mathrm{Mb}$ and especially $\mathrm{DroHb}$.
Figure 3 shows the compositions of the principal EM along the protein structure for the three studied cases in the most relevant coordination states at three different temperatures: DroHb 5c and 6c at 300, 349, and $363 \mathrm{~K} ; \mathrm{Mb} 5 \mathrm{c}$ at 300, 354, and $363 \mathrm{~K}$; and $\mathrm{Ngb} 6 \mathrm{c}$ at 300,363 , and $373 \mathrm{~K}$. The results are consistent with the RMSF profiles.

As can be seen in Figure 3A,B, the principal EM sampled along the $\mathrm{MD}$ simulation for $\mathrm{DroHb}$ at room temperature includes the DE region in the $5 c$ state and the $F, G$, and $H$ helices in the $6 c$ state. However, at high temperature, the principal contribution to the first essential mode consists of the movement of the C-terminal region, which even masks the movements observed at room temperature, suggesting the onset of the denaturation process.

In the $\mathrm{Mb}$ case (Figure $3 \mathrm{C}$ ), the principal essential mode shows mainly contributions of the C-terminal extreme of the $\mathrm{G}$ helix and the GH loop. The increase in temperature induces an increase in the mobility of different areas such as the CD region and the $\mathrm{F}$ helix but not of terminal regions as was found in the case of DroHb.

$\mathrm{Ngb}$, the one with the highest experimental thermostability, shows a differential behavior. At room temperature, the principal essential mode is concentrated in the flexible areas (as observed in the RMSF calculations shown in Figure S6), which consist of the $\mathrm{CD}$ region and the $\mathrm{N}$ - and $\mathrm{C}$-terminal regions (Figure 3D). The increase in temperature leads to an increase in the fluctuations of the $\mathrm{CD}$ region, as observed in the increase of the $\mathrm{CD}$ region contribution to the essential mode. Importantly, the principal EM is not significantly modified at high temperature, which indicates that the rise in temperature produces only an increase in the mobility of already flexible regions. 


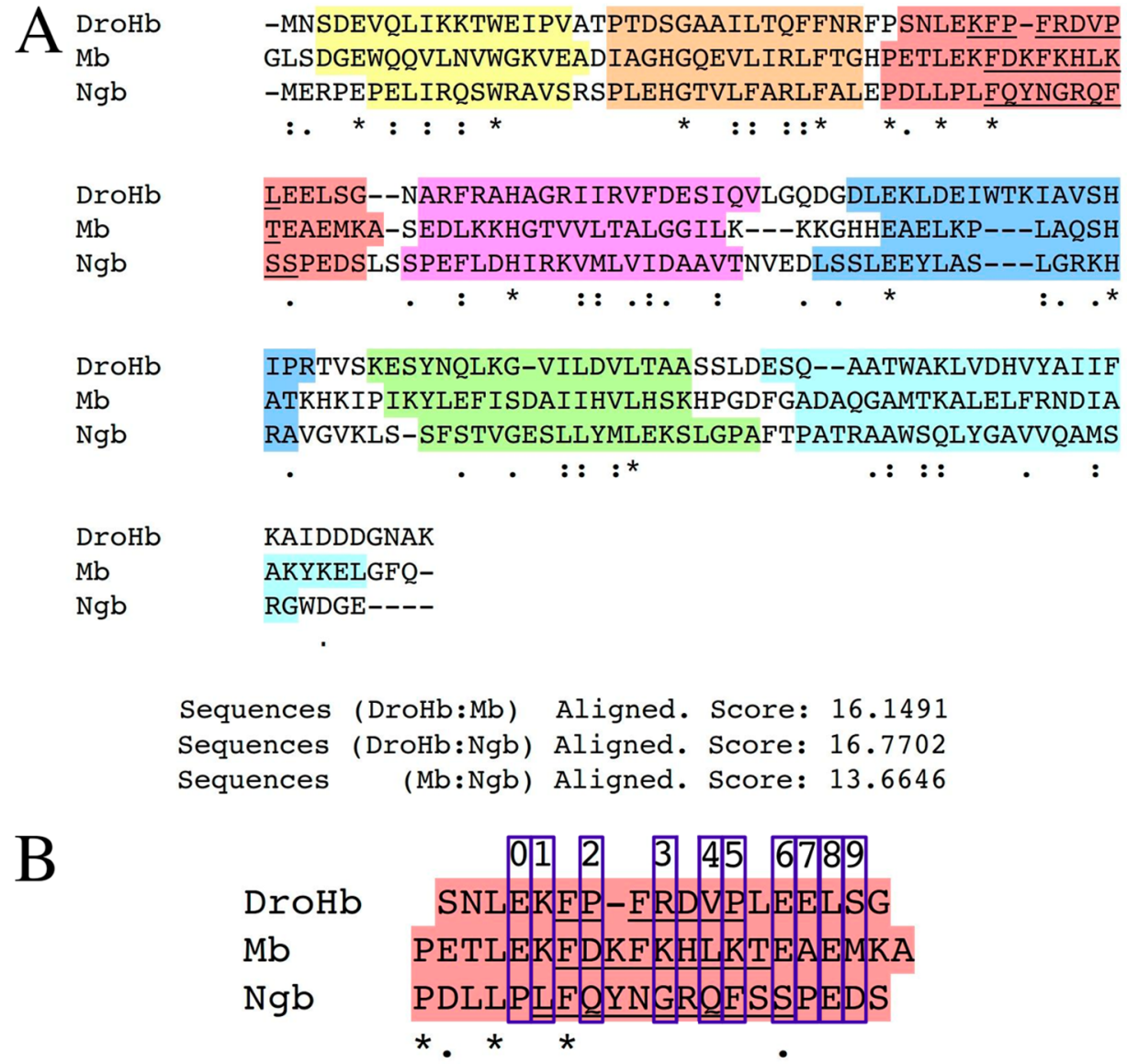

Figure 5. (A) Multiple sequence alignment of the studied proteins and corresponding alignment scores. The sequence is colored as in Figure 1: helix A (yellow), helix B (orange), CD region (red, with the CD loop underlined), helix E (fuchsia), helix F (blue), helix G (green), and helix H (cyan). (B) Closer look at the CD region. Some residues are marked for easy reference. Sequence alignment was carried out using Clustal $\mathrm{W}^{51}$ provided by GenomeNet (http://www.genome.jp).

In order to complete the analysis of the thermal effects on protein mobility, we calculated the conformational entropy (Table S5) for each case using the Andricioaei-Karplus approach $^{50}$ (details on the calculations are given in the Supporting Information). As expected, the conformational entropy increases with increasing temperature. The largest change is observed in DroHb, but it becomes similar to the rest of the cases when the protein $\mathrm{N}$ - and $\mathrm{C}$-terminal regions are not considered in the calculation. This is consistent with the changes found in the fluctuations of these regions (see Figure 2 and Table 2). Additionally, $\mathrm{Mb} 5 \mathrm{c}$ presents an important contribution of the $\mathrm{CD}$ region to the increase in the conformational entropy with increasing temperature. In $\mathrm{Ngb}$, on the other hand, this is observed in the $5 \mathrm{c}$ state, while in the $6 c$ state the variation is almost maintained when we exclude the $\mathrm{CD}$ region. From our previous analysis, we know that the $\mathrm{CD}$ region is more rigid in $\mathrm{Mb}$ than in $\mathrm{Ngb}$. Thus, an increase in the total entropy of this region will probably introduce structural instability, in contrast with $\mathrm{Ngb}$, where this mobility can be accommodated more easily.

Finally, in order to characterize the effect of hightemperature conditions on the tertiary structure and the propensity of each protein to lose its native structure with an increase in temperature, we calculated the evolution of the total number of native contacts along the simulations (Figure S8). In a first step, all of the contacts (independent of the nature of the amino acids involved) were considered. In the
$\mathrm{Ngb} 6 \mathrm{c}$ case, the number of native contacts remains stable along the simulation, even at high temperature. In Mb 5c, at room temperature the number of contacts along the $\mathrm{MD}$ simulation is also maintained, but at high temperature this value is significantly decreased. Similar behavior is observed in $\mathrm{DroHb}$, with an even more abrupt loss of native contacts after $500 \mathrm{~ns}$ at high temperature. Interestingly, Ngb has originally the lowest number of total contacts but is able to preserve this number when the temperature is increased.

To gain further insight, this analysis was performed separately for hydrophobic and hydrophilic contacts (Table $\mathrm{S6}$ ). Interestingly, the results indicate that $\mathrm{Ngb} 6 \mathrm{c}$ displays the highest number of hydrophobic contacts. On the other hand, $\mathrm{DroHb}$ displays a slightly lower number of hydrophobic contacts than $\mathrm{Ngb}$ but a higher number of hydrophilic contacts. At high temperature, the percentages of lost hydrophobic and hydrophilic contacts are significantly higher in DroHb than in $\mathrm{Ngb}$. In the case of $\mathrm{Mb}$, the total number of contacts is lower than in $\mathrm{Ngb}$ and $\mathrm{DroHb}$, but the percentage of contacts that are lost at high temperature is intermediate with respect to the values obtained in the other two proteins.

The regions where the different proteins lose native contacts at high temperature can be identified with differential contact maps, which are shown in Figure 4. In Ngb 6c, the loss of native contacts is concentrated in the $\mathrm{CD}$ region. In $\mathrm{Mb} 5 \mathrm{c}$, the lost contacts correspond to the $\mathrm{CD}$ and $\mathrm{EF}$ regions. On the other hand, DroHb 5c also loses contacts in the EF region but 
Ai) DroHb 5c 300K

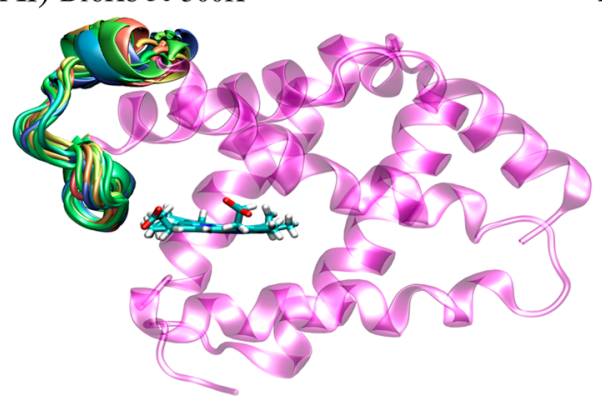

Bi) $\mathrm{Mb} 5 \mathrm{c} 300 \mathrm{~K}$

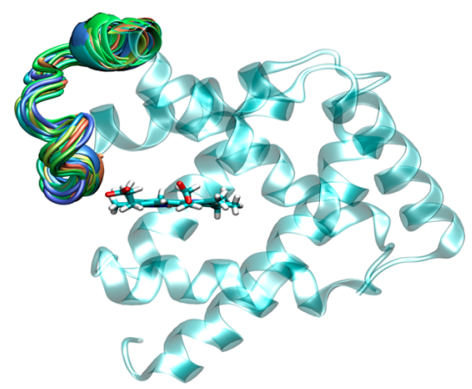

Ci) Ngb 5c 300K

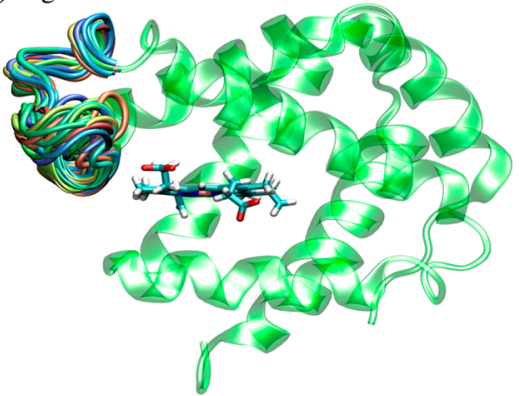

Aii) DroHb 6c 300K

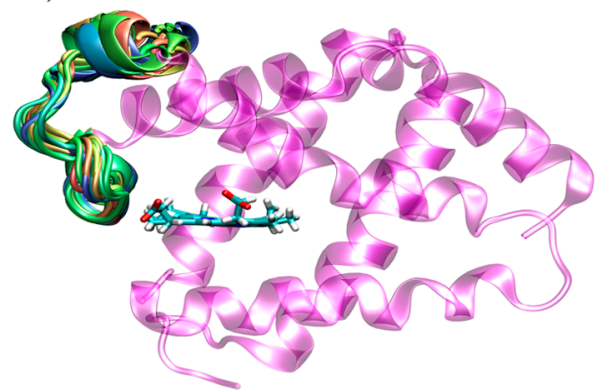

Bii) $\mathrm{Mb} 6 \mathrm{c} 300 \mathrm{~K}$

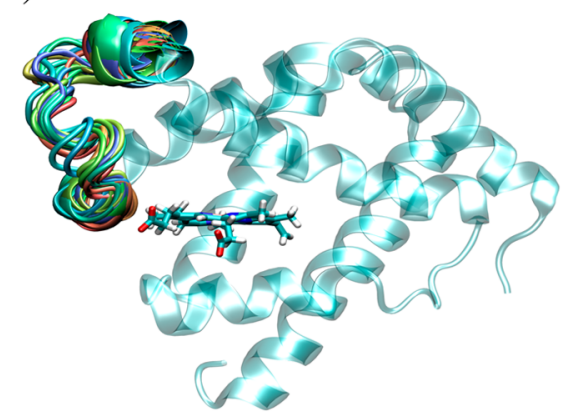

Cii) Ngb 6c 300K

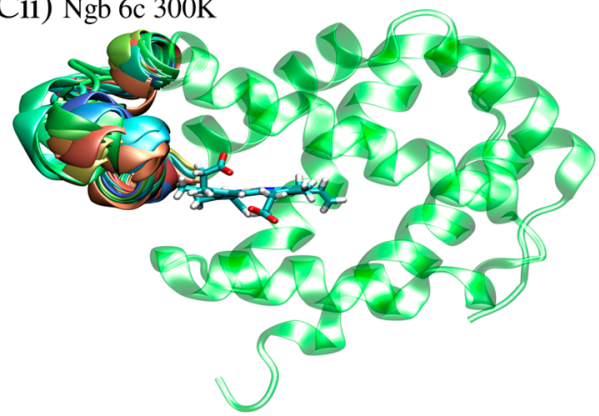

Figure 6. Superposition of snapshots along the MD trajectories at $300 \mathrm{~K}$ : (Ai) DroHb 5c, (Aii) DroHb 6c, (Bi) Mb 5c, (Bii) Mb 6c, (Ci) Ngb 5c, and (Cii) Ngb 6c. Trajectory alignment in this case was done excluding the $\mathrm{CD}$ region, which is the only protein region that is shown repeatedly for clarity purposes.

more importantly, contacts are lost in the C-terminal extreme as well. The loss of contacts in DroHb $6 \mathrm{c}$ is found in the GH region. This indicates that while Ngb loses interactions only in the $\mathrm{CD}$ region, which is already poorly structured, $\mathrm{DroHb}$ loses native contacts in regions that are structured at room temperature.

Finally, heme-protein contacts were also analyzed and compared for the different temperature conditions. No significant differences in the time scale of the simulations were observed (data not shown). Along the simulations, the heme group remains close to its initial position in all cases. As shown in Figure S9, under high-temperature conditions the fluctuations of the heme side chains are slightly increased, but globally the location of the heme group in the protein matrix remains unchanged.

In order to further understand the differences in the flexibilities of certain regions observed for the different proteins, we aligned and compared their sequences (Figure $5)$. At this point, the $\mathrm{CD}$ region was compared as a whole (including the $\mathrm{C}$ and $\mathrm{D}$ helices and the $\mathrm{CD}$ loop) and considering only the CD loop itself (underlined in Figure 5B). $\mathrm{Ngb}$ presents the shortest A and G helices of the three proteins but has the largest $\mathrm{CD}$ loop and $\mathrm{CD}$ region. $\mathrm{Mb}$ has the largest
$\mathrm{A}$ and $\mathrm{H}$ helices, and finally, DroHb has the largest $\mathrm{F}$ helix and the shortest CD loop.

Focusing on the $\mathrm{CD}$ region sequence enables the identification of some residue positions that might influence the inherent flexibility of the $C D$ region. These positions are marked in Figure 5B with numbers 0 to 9 . In $\mathrm{Ngb}$, position \#0 and position \#7, which are occupied by Pro, delimit the CD loop since they mark the end of the $C$ helix and the beginning of the $\mathrm{D}$ helix, respectively. This results in a larger CD loop for $\mathrm{Ngb}$ in comparison with the other two cases, where both helices extend inside this part of the sequence. In position \#1, $\mathrm{Ngb}$ displays a Leu residue, while $\mathrm{DroHb}$ and $\mathrm{Mb}$ present a Lys residue. Additionally, $\mathrm{DroHb}$ shows the presence of two Pro residues in the middle of the CD loop (at positions \#2 and \#5), which probably contribute to rigidification of this region. Another interesting observation can be made regarding positions \#3, \#4, \#6, and \#8, where $\mathrm{Mb}$ and DroHb share similar residues while $\mathrm{Ngb}$ presents different amino acids. In position \#3, Ngb displays a Gly residue, which is a flexible residue, whereas $\mathrm{Mb}$ and $\mathrm{DroHb}$ exhibit basic residues (Lys and Arg, respectively). In position \#4, $\mathrm{DroHb}$ and $\mathrm{Mb}$ have Val and Leu residues, respectively, but $\mathrm{Ngb}$ has a polar residue (Gln). In position \#6, DroHb and $\mathrm{Mb}$ both have a Glu residue, which is replaced by Ser in $\mathrm{Ngb}$. In position \#8, DroHb has a 
Leu residue while $\mathrm{Mb}$ and $\mathrm{Ngb}$ have an acidic residue (Glu). Finally, in position \#9, a Ser residue in $\mathrm{DroHb}$ is replaced by Met in $\mathrm{Mb}$ and Asp in Ngb. All of these data indicate that $\mathrm{DroHb}$ and $\mathrm{Mb}$ contain $\mathrm{CD}$ loops that are more similar in sequence (and probably in their dynamical properties) with respect to $\mathrm{Ngb}$. To support this information, the conformational variation of the $\mathrm{CD}$ region along $\mathrm{MD}$ simulations at 300 $\mathrm{K}$ is shown in Figure 6. Clearly, Ngb ( $\mathrm{Ci}$ for $5 \mathrm{c}$ and Cii for $6 \mathrm{c}$ ) explores a wider region of space than $\mathrm{DroHb}$ ( $\mathrm{Ai}$ for $5 \mathrm{c}$ and Aii for $6 \mathrm{c}$ ) and $\mathrm{Mb}$ (Bi for $5 \mathrm{c}$ and Bii for $6 \mathrm{c}$ ). Finally, although this analysis confirms the fact that the $\mathrm{CD}$ region of $\mathrm{Ngb}$ differs from those of $\mathrm{Mb}$ and $\mathrm{DroHb}$ both in sequence and in dynamical properties, the question of the molecular mechanism of how these specific changes in sequence result in higher flexibility is still open.

\section{DISCUSSION}

Differential thermostability in globins has been related in the past with the existence of strong interactions, protein flexibility, and hexacoordination, among other structural and dynamical factors. $^{9-11,52-54}$ Many theoretical works, mainly based on MD simulations, were focused on the thermostability of proteins belonging to thermophilic organisms as well as proteins developed by directed evolution. The conclusions are strongly dependent on the analyzed system. In some cases, higher protein flexibility is observed in thermostable proteins with regard to their mesophilic homologues. ${ }^{48,49,52}$ Additionally, an increase in the low-frequency movements was observed in thermostable proteins, which was related to an increase in the conformational entropy of the native state, thus providing a higher stability to the native structure. ${ }^{48}$ However, other studies found that thermophilic variants display higher rigidity than their mesophilic homologues. ${ }^{15,22}$ Many experimental works also highlighted the difficulty of establishing general criteria for the requirements to increase the thermal stability of proteins. In this context, many experimental results challenge the idea that enhanced stability is achieved in the context of stronger interactions and higher rigidity. ${ }^{7,9,55}$

In this work, we intended, by means of MD simulations, to shed light on the molecular basis of thermostability in globins, taking as representative examples $\mathrm{DroHb}$, horse heart $\mathrm{Mb}$, and human brain $\mathrm{Ngb}$, due to the differences in $T_{\mathrm{m}}$ as well as in the populations of the penta- and hexacoordinated states. Special attention has been paid to the $\mathrm{CD}$ region, which has been reported in previous works to be a key region for hexacoordination and thermostability, ${ }^{5,7}$ among other biophysical processes.

Globally, the stabilities of the different proteins at high temperature can be observed comparing the total RMSDs along the MD trajectories with respect to the native (crystal) structure. While Ngb RMSD values remain almost invariant at high temperature, DroHb displays a significant increase in the RMSD under high-temperature conditions. For $\mathrm{Mb}$, there is also an increase in the RMSD value when the temperature is raised, but not as large as that in DroHb. This is consistent with the experimental trend observed for the $T_{\mathrm{m}}$ values of the three proteins. $^{25}$

In regard to the protein mobility, our results indicate that $\mathrm{DroHb}$ is the protein with the stiffest $\mathrm{CD}$ region, whereas $\mathrm{Ngb}$ has the most flexible one. On the other hand, DroHb displays the largest increase in the mobility of the C-terminal extreme with the increase in temperature. The $\mathrm{C}$ - and $\mathrm{N}$-termini of $\mathrm{Mb}$ and $\mathrm{Ngb}$ are already flexible at room temperature, and when the temperature is increased, the fluctuations remain similar in $\mathrm{Ngb}$ and slightly increase in $\mathrm{Mb}$. This is also confirmed by the $\mathrm{ED}$ analysis, where DroHb shows an increase in the mobility of the C-terminus in both coordination states at high temperature. For Ngb, the essential modes do not show significant differences with increasing temperature, and the $\mathrm{CD}$ region is the most affected structural element. Finally, the analysis of native contacts along the $\mathrm{MD}$ simulations shows that they are much more conserved in $\mathrm{Ngb}$ than in $\mathrm{Mb}$ and $\mathrm{DroHb}$ when the temperature is increased. The loss of native contacts in $\mathrm{Ngb}$ is concentrated in the $\mathrm{CD}$ region, while in the rest of the cases it is expanded along the protein sequence.

When all of these data are considered, questions arise concerning what is the key factor that governs the thermal stability in globins and, in particular, how Ngb manages to conserve the tertiary structure and avoid the movement of intrinsically rigid regions. In this context, two possible and not mutually exclusive hypotheses can be made: (i) the observed low mobility of the protein core at high temperature can originate from a strong combination of polar and hydrophobic interactions, and (ii) the existence of a very flexible region (the $\mathrm{CD}$ region) stabilizes the native state by increasing the conformational entropy of the native state and providing available nondenaturating protein movements.

Additionally, in previous works, protein flexibility in globins has also been associated with the coordination state of the heme. ${ }^{6,7,30,56}$ However our results indicate that the increased thermostability in $\mathrm{Ngb}$ with respect to $\mathrm{Mb}$ is not directly associated with the fact that $\mathrm{Ngb}$ is a hexacoordinated protein but rather is due to the intrinsic flexibility of the Ngb CD region, which in turn favors the formation of the hexacoordinated state. Moreover, as mentioned in the Introduction, the CO-bound form of $\mathrm{Ngb}$ displays a $T_{\mathrm{m}}$ lower than that of $\mathrm{Ngb}$ $6 \mathrm{c}$ but still higher than those of $\mathrm{Mb}$ and DroHb. ${ }^{25}$ Considering these results, it may be deduced that higher flexibility of the $\mathrm{CD}$ region is a key factor, but probably not exclusive, in controlling the thermostability. Altogether, our results indicate that $\mathrm{Ngb}$ is able to combine a strong and stable protein core, characterized by strong and stable native contacts, with a highly flexible $\mathrm{CD}$ region, resulting in an extremely stable globin.

\section{CONCLUSIONS}

The aim of this study was to shed light on the different structural and dynamical mechanisms that govern thermostability in globins by means of molecular dynamics simulations at different temperatures. Our theoretical results indicate a close relationship between the flexibility of the CD region and its thermostability. The MD simulations showed that at high temperature a major loss of tertiary structure is observed for DroHb, which may facilitate the denaturation process increasing the movement of the $\mathrm{N}$ - and C-terminal extremes. On the other hand, Ngb, which displays the highest thermostability of the three proteins studied, is able to keep its tertiary structure by increasing the movement of very flexible regions of the protein, especially the $\mathrm{CD}$ region, while keeping the strong contact network in the rest of the protein structure. The results of this work highlight the importance of flexibility in thermal stability as well as the key role of the $\mathrm{CD}$ region in globins, which is found to be very relevant for determining their dynamics, biochemistry, and stability. 


\section{ASSOCIATED CONTENT}

\section{S Supporting Information}

The Supporting Information is available free of charge on the ACS Publications website at DOI: 10.1021/acs.jcim.8b00840.

Superposition of initial X-ray structures, validation of the applied water model at high temperature, RMSD along the MD simulations, analysis of the replica $\mathrm{MD}$ simulations, additional information on the essential dynamics analysis, conformational entropy calculations, analysis of native contacts along the MD simulations, and positional fluctuation of the heme group in the MD simulations at room and high temperature (PDF)

\section{AUTHOR INFORMATION}

\section{Corresponding Author}

*E-mail: lula@qi.fcen.uba.ar.

\section{ORCID}

Dario A. Estrin: 0000-0002-5006-7225

F. Javier Luque: 0000-0002-8049-3567

Luciana Capece: 0000-0003-3794-5713

Notes

The authors declare no competing financial interest.

\section{ACKNOWLEDGMENTS}

This research was supported by grants from the Universidad de Buenos Aires (UBACYT 20020120300025BA), Agencia Nacional de Promoción Científica y Tecnológica (PICT 2012-2571, PICT 2014-1022, and PICT 2015-2761), CONICET (Grants $11220150100303 \mathrm{CO}$ and PIP201211220130100542), ANPCYT (PICT 2013-1417), Spanish Maria de Maeztu program (MDM-2017-0767) and Generalitat de Catalunya (2017SGR1746). We acknowledge CECAR and the Barcelona Supercomputer Center (BCV2016-3-0015) for providing access to computation resources. L.J.P. holds a CONICET Ph.D. fellowship. D.A.E. and L.C. are members of CONICET.

\section{REFERENCES}

(1) Burmester, T.; Weich, B.; Reinhardt, S.; Hankeln, T. A Vertebrate Globin Expressed in the Brain. Nature 2000, 407, 520523.

(2) Vinogradov, S. N.; Hoogewijs, D.; Bailly, X.; Arredondo-Peter, R.; Guertin, M.; Gough, J.; Dewilde, S.; Moens, L.; Vanfleteren, J. R. Three Globin Lineages Belonging to Two Structural Classes in Genomes from the Three Kingdoms of Life. Proc. Natl. Acad. Sci. U. S. A. 2005, 102, 11385-11389.

(3) Vinogradov, S. N.; Hoogewijs, D.; Bailly, X.; Arredondo-Peter, R.; Gough, J.; Dewilde, S.; Moens, L.; Vanfleteren, J. R. A Phylogenomic Profile of Globins. BMC Evol. Biol. 2006, 6, 31.

(4) Jain, R.; Chan, M. K. Mechanisms of Ligand Discrimination by Heme Proteins. JBIC, J. Biol. Inorg. Chem. 2003, 8, 1-11.

(5) Bustamante, J. P.; Radusky, L.; Boechi, L.; Estrin, D. A.; ten Have, A.; Martí, M. A. Evolutionary and Functional Relationships in the Truncated Hemoglobin Family. PLoS Comput. Biol. 2016, 12, e1004701.

(6) Capece, L.; Marti, M. A.; Bidon-Chanal, A.; Nadra, A.; Luque, F. J.; Estrin, D. A. High Pressure Reveals Structural Determinants for Globin Hexacoordination: Neuroglobin and Myoglobin Cases. Proteins: Struct., Funct., Genet. 2009, 75, 885-894.

(7) Boron, I.; Capece, L.; Pennacchietti, F.; Wetzler, D. E.; Bruno, S.; Abbruzzetti, S.; Chisari, L.; Luque, F. J.; Viappiani, C.; Marti, M. A.; Estrin, D. A.; Nadra, A. D. Engineered Chimeras Reveal the Structural Basis of Hexacoordination in Globins: A Case Study of
Neuroglobin and Myoglobin. Biochim. Biophys. Acta, Gen. Subj. 2015, $1850,169-177$.

(8) Morzan, U. N.; Capece, L.; Marti, M. A.; Estrin, D. A. Quaternary Structure Effects on the Hexacoordination Equilibrium in Rice Hemoglobin RHb1: Insights from Molecular Dynamics Simulations. Proteins: Struct., Funct., Genet. 2013, 81, 863-873.

(9) Chen, L.; Roberts, M. F. Characterization of a Tetrameric Inositol Monophosphatase from the Hyperthermophilic Bacterium Thermotoga Maritima. Appl. Environ. Microbiol. 1999, 65, 45594567.

(10) Auerbach, G.; Ostendorp, R.; Prade, L.; Korndörfer, I.; Dams, T.; Huber, R.; Jaenicke, R. Lactate Dehydrogenase from the Hyperthermophilic Bacterium Thermotoga Maritima: The Crystal Structure at $2.1 \AA$ Resolution Reveals Strategies for Intrinsic Protein Stabilization. Structure 1998, 6, 769-781.

(11) Vieille, C.; Hess, J. M.; Kelly, R. M.; Zeikus, J. G. XylA Cloning and Sequencing and Biochemical Characterization of Xylose Isomerase from Thermotoga Neapolitana. Appl. Environ. Microbiol. 1995, 61, $1867-1875$.

(12) Donati, E. R.; Castro, C.; Urbieta, M. S. Thermophilic Microorganisms in Biomining. World J. Microbiol. Biotechnol. 2016, 32, 179.

(13) Zeldes, B. M.; Keller, M. W.; Loder, A. J.; Straub, C. T.; Adams, M. W. W.; Kelly, R. M. Extremely Thermophilic Microorganisms as Metabolic Engineering Platforms for Production of Fuels and Industrial Chemicals. Front. Microbiol. 2015, 6, 1209.

(14) Bryngelson, J. D.; Onuchic, J. N.; Socci, N. D.; Wolynes, P. G. Funnels, Pathways, and the Energy Landscape of Protein Folding: A Synthesis. Proteins: Struct., Funct., Genet. 1995, 21, 167-195.

(15) Vogt, G.; Woell, S.; Argos, P. Protein Thermal Stability, Hydrogen Bonds, and Ion Pairs. J. Mol. Biol. 1997, 269, 631-643.

(16) Matthews, B. W.; Nicholson, H.; Becktel, W. J. Enhanced Protein Thermostability from Site-Directed Mutations That Decrease the Entropy of Unfolding. Proc. Natl. Acad. Sci. U. S. A. 1987, 84, 6663-6667.

(17) Tang, K. E. S.; Dill, K. A. Native Protein Fluctuations: The Conformational-Motion Temperature and the Inverse Correlation of Protein Flexibility with Protein Stability. J. Biomol. Struct. Dyn. 1998, 16, 397-411.

(18) Mamonova, T. B.; Glyakina, A. V.; Galzitskaya, O. V.; Kurnikova, M. G. Stability and Rigidity/Flexibility-Two Sides of the Same Coin? Biochim. Biophys. Acta, Proteins Proteomics 2013, 1834, 854-866.

(19) Manco, G.; Giosuè, E.; D’Auria, S.; Herman, P.; Carrea, G.; Rossi, M. Cloning, Overexpression, and Properties of a New Thermophilic and Thermostable Esterase with Sequence Similarity to Hormone-Sensitive Lipase Subfamily from the Archaeon Archaeoglobus fulgidus. Arch. Biochem. Biophys. 2000, 373, 182-192.

(20) Gershenson, A.; Schauerte, J. A.; Giver, L.; Arnold, F. H. Tryptophan Phosphorescence Study of Enzyme Flexibility and Unfolding in Laboratory-Evolved Thermostable Esterases. Biochemistry 2000, 39, 4658-4665.

(21) Závodszky, P.; Kardos, J.; Svingor, Á.; Petsko, G. A. Adjustment of Conformational Flexibility Is a Key Event in the Thermal Adaptation of Proteins. Proc. Natl. Acad. Sci. U. S. A. 1998, 95, $7406-7411$

(22) Lazaridis, T.; Lee, I.; Karplus, M. Dynamics and Unfolding Pathways of a Hyperthermophilic and a Mesophilic Rubredoxin. Protein Sci. 1997, 6, 2589-2605.

(23) Quezada, A. G.; Díaz-Salazar, A. J.; Cabrera, N.; PérezMontfort, R.; Piñeiro, A.; Costas, M. Interplay between Protein Thermal Flexibility and Kinetic Stability. Structure 2017, 25, 167179.

(24) Bustamante, J. P.; Bonamore, A.; Nadra, A. D.; Sciamanna, N.; Boffi, A.; Estrin, D. A.; Boechi, L. Molecular Basis of Thermal Stability in Truncated (2/2) Hemoglobins. Biochim. Biophys. Acta, Gen. Subj. 2014, 1840, 2281-2288. 
(25) Hamdane, D.; Kiger, L.; Dewilde, S.; Uzan, J.; Burmester, T.; Hankeln, T.; Moens, L.; Marden, M. C. Hyperthermal Stability of Neuroglobin and Cytoglobin. FEBS J. 2005, 272, 2076-2084.

(26) De Sanctis, D.; Dewilde, S.; Vonrhein, C.; Pesce, A.; Moens, L.; Ascenzi, P.; Hankeln, T.; Burmester, T.; Ponassi, M.; Nardini, M.; Bolognesi, M. Bishistidyl Heme Hexacoordination, a Key Structural Property in Drosophila Melanogaster Hemoglobin. J. Biol. Chem. 2005, 280, 27222-27229.

(27) Barends, T. R. M.; Foucar, L.; Ardevol, A.; Nass, K.; Aquila, A.; Botha, S.; Doak, R. B.; Falahati, K.; Hartmann, E.; Hilpert, M.; Heinz, M.; Hoffmann, M. C.; Köfinger, J.; Koglin, J. E.; Kovacsova, G.; Liang, M.; Milathianaki, D.; Lemke, H. T.; Reinstein, J.; Roome, C. M.; Shoeman, R. L.; Williams, G. J.; Burghardt, I.; Hummer, G.; Boutet, S.; Schlichting, I. Direct Observation of Ultrafast Collective Motions in CO Myoglobin upon Ligand Dissociation. Science 2015, 350, 445450.

(28) Pesce, A.; Dewilde, S.; Nardini, M.; Moens, L.; Ascenzi, P.; Hankeln, T.; Burmester, T.; Bolognesi, M. Human Brain Neuroglobin Structure Reveals a Distinct Mode of Controlling Oxygen Affinity. Structure 2003, 11, 1087-1095.

(29) Arroyo Mañez, P.; Lu, C.; Boechi, L.; Martí, M. A.; Shepherd, M.; Wilson, J. L.; Poole, R. K.; Luque, F. J.; Yeh, S. R.; Estrin, D. A. Role of the Distal Hydrogen-Bonding Network in Regulating Oxygen Affinity in the Truncated Hemoglobin III from Campylobacter Jejuni. Biochemistry 2011, 50, 3946-3956.

(30) Capece, L.; Marti, M. A.; Crespo, A.; Doctorovich, F.; Estrin, D. A. Heme Protein Oxygen Affinity Regulation Exerted by Proximal Effects. J. Am. Chem. Soc. 2006, 128, 12455-12461.

(31) Case, D. A.; Babin, V.; Berryman, J. T.; Betz, R. M.; Cai, Q.; Cerutti, D. S.; Cheatham, T. E., III; Darden, T. A.; Duke, R. E.; Gohlke, H.; Goetz, A. W.; Gusarov, S.; Homeyer, N.; Janowski, P.; Kaus, J.; Kolossváry, I.; Kovalenko, A.; Lee, T. S.; LeGrand, S.; Luchko, T.; Luo, R.; Madej, B.; Merz, K. M.; Paesani, F.; Roe, D. R.; Roitberg, A.; Sagui, C.; Salomon-Ferrer, R.; Seabra, G.; Simmerling, C. L.; Smith, W.; Swails, J.; Walker, R. C.; Wang, J.; Wolf, R. M.; Wu, X.; Kollman, P. A. AMBER 14; University of California: San Francisco, 2014.

(32) Jorgensen, W. L.; Chandrasekhar, J.; Madura, J. D.; Impey, R. W.; Klein, M. L. Comparison of Simple Potential Functions for Simulating Liquid Water. J. Chem. Phys. 1983, 79, 926-935.

(33) Marti, M. A.; Crespo, A.; Capece, L.; Boechi, L.; Bikiel, D. E. D. E.; Scherlis, D. A. D. A.; Estrin, D. A. Dioxygen Affinity in Heme Proteins Investigated by Computer Simulation. J. Inorg. Biochem. 2006, 100, 761-770.

(34) Marti, M. A.; Capece, L.; Bidon-Chanal, A.; Crespo, A.; Guallar, V.; Luque, F. J.; Estrin, D. A. Nitric Oxide Reactivity with Globins as Investigated Through Computer Simulation. Methods Enzymol. 2008, 437, 477-498.

(35) Bikiel, D. E.; Boechi, L.; Capece, L.; Crespo, A.; De Biase, P. M.; Di Lella, S.; González Lebrero, M. C.; Marti, M. A.; Nadra, A. D.; Perissinotti, L. L.; Scherlis, D. A.; Estrin, D. A. Modeling Heme Proteins Using Atomistic Simulations. Phys. Chem. Chem. Phys. 2006, $8,5611-5628$.

(36) Perissinotti, L. L.; Marti, M. A.; Doctorovich, F.; Luque, F. J.; Estrin, D. A. A Microscopic Study of the Deoxyhemoglobin-Catalyzed Generation of Nitric Oxide from Nitrite Anion. Biochemistry 2008, 47, 9793-9802.

(37) Giordano, D.; Boechi, L.; Vergara, A.; Martí, M. A.; Samuni, U.; Dantsker, D.; Grassi, L.; Estrin, D. A.; Friedman, J. M.; Mazzarella, L.; Di Prisco, G.; Verde, C. The Hemoglobins of the Sub-Antarctic Fish Cottoperca Gobio, a Phyletically Basal Species - Oxygen-Binding Equilibria, Kinetics and Molecular Dynamics. FEBS J. 2009, 276, 2266-2277.

(38) Capece, L.; Lewis-Ballester, A.; Marti, M. A.; Estrin, D. A.; Yeh, S. R. Molecular Basis for the Substrate Stereoselectivity in Tryptophan Dioxygenase. Biochemistry 2011, 50, 10910-10918.

(39) Forti, F.; Boechi, L.; Bikiel, D.; Martí, M. A.; Nardini, M.; Bolognesi, M.; Viappiani, C.; Estrin, D.; Luque, F. J. Ligand Migration in Methanosarcina Acetivorans Protoglobin: Effects of Ligand Binding and Dimeric Assembly. J. Phys. Chem. B 2011, 115, 13771-13780.

(40) Ryckaert, J.-P.; Ciccotti, G.; Berendsen, H. J. C. Numerical Integration of the Cartesian Equations of Motion of a System with Constraints: Molecular Dynamics of n-Alkanes. J. Comput. Phys. 1977, 23, 327-341.

(41) Berendsen, H. J. C.; Postma, J. P. M.; van Gunsteren, W. F.; DiNola, A.; Haak, J. R. Molecular Dynamics with Coupling to an External Bath. J. Chem. Phys. 1984, 81, 3684-3690.

(42) Wu, X.; Brooks, B. R.; Vanden-Eijnden, E. Self-Guided Langevin Dynamics via Generalized Langevin Equation. J. Comput. Chem. 2016, 37, 595-601.

(43) Humphrey, W.; Dalke, A.; Schulten, K. VMD: Visual Molecular Dynamics. J. Mol. Graphics 1996, 14, 33-38.

(44) Amadei, A.; Linssen, A. B. M.; Berendsen, H. J. C. Essential Dynamics of Proteins. Proteins: Struct., Funct., Genet. 1993, 17, 412425.

(45) Nadra, A. D.; Martí, M. A.; Pesce, A.; Bolognesi, M.; Estrin, D. A. Exploring the Molecular Basis of Heme Coordination in Human Neuroglobin. Proteins: Struct., Funct., Genet. 2008, 71, 695-705.

(46) Bidon-Chanal, A.; Martí, M. A.; Crespo, A.; Milani, M.; Orozco, M.; Bolognesi, M.; Luque, F. J.; Estrin, D. A. Ligand-Induced Dynamical Regulation of NO Conversion in Mycobacterium Tuberculosis Truncated Hemoglobin-N. Proteins: Struct., Funct., Genet. 2006, 64, 457-464.

(47) Trent, J. T.; Watts, R. A.; Hargrove, M. S. Human Neuroglobin, a Hexacoordinate Hemoglobin That Reversibly Binds Oxygen. J. Biol. Chem. 2001, 276, 30106-30110.

(48) Wintrode, P. L.; Zhang, D.; Vaidehi, N.; Arnold, F. H.; Goddard, W. A. Protein Dynamics in a Family of Laboratory Evolved Thermophilic Enzymes. J. Mol. Biol. 2003, 327, 745-757.

(49) Colombo, G.; Merz, K. M. Stability and Activity of Mesophilic Subtilisin E and Its Thermophilic Homolog: Insights from Molecular Dynamics Simulations. J. Am. Chem. Soc. 1999, 121, 6895-6903.

(50) Andricioaei, I.; Karplus, M. On the Calculation of Entropy from Covariance Matrices of the Atomic Fluctuations. J. Chem. Phys. 2001, $115,6289-6292$.

(51) Thompson, J. D.; Higgins, D. G.; Gibson, T. J. CLUSTAL W: Improving the Sensitivity of Progressive Multiple Sequence Alignment through Sequence Weighting, Position-Specific Gap Penalties and Weight Matrix Choice. Nucleic Acids Res. 1994, 22, 4673-4680.

(52) Jaenicke, R.; Böhm, G. The Stability of Proteins in Extreme Environments. Curr. Opin. Struct. Biol. 1998, 8, 738-748.

(53) Adams, M. W. W.; Kelly, R. M. Enzymes from Microorganisms in Extreme Environments. Chem. Eng. News 1995, 73, 32-42.

(54) Jaenicke, R. Protein Stability and Molecular Adaptation to Extreme Conditions. Eur. J. Biochem. 1991, 202, 715-728.

(55) Hernandez, G.; Jenney, F. E.; Adams, M. W. W.; LeMaster, D. M. Millisecond Time Scale Conformational Flexibility in a Hyperthermophile Protein at Ambient Temperature. Proc. Natl. Acad. Sci. U. S. A. 2000, 97, 3166-3170.

(56) Martí, M. A.; Capece, L.; Bikiel, D. E.; Falcone, B.; Estrin, D. A. Oxygen Affinity Controlled by Dynamical Distal Conformations: The Soybean Leghemoglobin and the Paramecium Caudatum Hemoglobin Cases. Proteins: Struct., Funct., Genet. 2007, 68, 480-487. 